\title{
L'EFFICACITÉ DES DROITS SOCIAUX EN SANTÉ PUBLIQUE
}

\author{
CARLOS NETO, Daniel ${ }^{1}$
}

DENDASCK, Carla ${ }^{2}$

OLIVEIRA, Euzébio de ${ }^{3}$

CARLOS NETO, Daniel; DENDASCK, Carla; OLIVEIRA, Euzébio de. L'efficacité des droits sociaux en santé publique. Connaissances de base scientifique multidisciplinaire Magazine - Vol. 1. An. 1. Mars. 2016, pp: 3-14 ISSN:0959-2448

\section{RÉSUMÉ}

Les droits fondamentaux ont été cause de diverses discussions ces dernières années, causant des érudits se consacrer pour étudier, que ce soit par l'aspect de la santé, comme par le biais de la Loi, l'efficacité réelle des lignes directrices établies par les organisations. Cet article vise à apporter une brève réflexion sur l'efficacité réelle des droits sociaux dans le brésilien de la santé publique, en utilisant comme base, une analyse exploratoire de l'elucidações par plusieurs chercheurs qui imprègnent pour corroborer cela. Apportant une brève discussion du minimum existentiel.

${ }^{1}$ Avocat. De la médecine universitaire. Doctorat en santé publique. Executive Mba. Spécialisée dans les services de santé appliquées. Remise des diplômes en santé familiale. Spécialiste de l'audit. Courriel : autor@nucleodoconhecimento.com.br 2 Doctorat en psychanalyse, post doctorant en psychanalyse clinique et maîtrise en bioéthique, directeur du Centre pour la recherche et le développement des études avancées, entraîneur et professeur de cours de MBA en compagnie, Campinas et São Paulo-email : autor@nucleodoconhecimento.com.br

${ }^{3}$ Biologiste. Master en biologie. Docteur en médecine tropicale maladies. Chercheur du programme diplômé du centre de médecine tropicale de l'UFPA. Professeur diplômé de troisième cycle et de niveau et chercheur à l'Université fédérale du Pará UFPA. Courriel : autor@nucleodoconhecimento.com.br 
Mots clés: Droits sociaux. Santé publique. Efficacité des droits fondamentaux.

\section{INTRODUCTION}

Droits fondamentaux peuvent être définies comme un ensemble de règles, les principes, les droits, les devoirs et les instituts impliqués dans la souveraineté populaire, qui garantissent la coexistence pacifique, libre et égale, quelle que soit la croyance, de race, origine, couleur, statut social ou la condition économique, tous basés sur le principe de la dignité humaine. (CELLULES SANGUINES, 2010)

Toutefois, à José Afonso da Silva, si conceptualiser des droits fondamentaux devient une tâche difficile devant les diverses transformations au fil du temps, voir :

L'expansion et la transformation des droits fondamentaux dans l'histoire qui rend difficile de définir un concept synthétique.

Elle augmente cette difficulté la circonstance d'employer des expressions différentes pour les désigner, tels que : droits naturels, droits de l'homme, droits de l'homme, droits individuels, publics droits subjectifs, des libertés fondamentales, libertés et droits fondamentaux et des droits de l'homme fondamentaux. (SILVA, 2005, p. 179)

Droits sociaux déjà, sont des droits fondamentaux de l'homme, comme élabore Moraes (2014, p. 595) :

Droits sociaux sont des droits fondamentaux de l'homme, parce qu'ils concernent un complexe des relations sociales, économiques ou culturels qui se développe pour la réalisation de la vie dans tout son potentiel, sans lequel le titulaire ne pouvait atteindre et apprécier les produits dont vous avez besoin.

II s'avère que la principale difficulté à surmonter dans cette rubrique fait référence à l'applicabilité et l'efficacité de ces droits, car comme nous le savons tous, la santé est positivada dans la liste des droits sociaux. 
Ainsi, il est important ici de mentionner la notion du terme "efficacité », comme l'exécution concrète de la fonction sociale du droit, comme suit :

L'idée de l'efficacité, à savoir, qui spécifie l'efficacité, correspond à cette Kelsendistinguer la notion d'efficacité de la norme-dépeint comme étant " la réalité de son efficacement et il a fait remarquer, le fait que procéder à un être humain comme la norme pour vérifier les faits ". L'efficacité signifie la réalisation du droit, l'exercice de sa fonction sociale. Elle représente la concrétisation, dans le monde des faits, les préceptes juridiques et symbolise le rapprochement, aussi près que possible, entre les niveaux normatif et doit être de la réalité sociale. (BARROSO, 2002, p. 236)

Quant à l'efficacité des droits sociaux est nécessaire à la compréhension non seulement de l'état de droit dans un sens large, mais la même approche avec d'autres systèmes, tels que : politique, économique, sociale et historique.

Il faut également la durée et l'efficacité de la norme, comme suit :

Le normativismo distingue, avec précision, la durée d'efficacité. La leçon de Kelsen est claire à cet égard. La durée de l'étalon, lui, appartient à la doit-être et ne pas à l'ordre de l'être. On entend que l'existence spécifie la norme ; l'efficacité réside dans le fait que la norme est effective et suivie ; la circonstance qu'une conduite humaine comme la norme s'applique aux faits. (SILVA, 2005, p. 64)

Une des questions plus importantes qui entourent l'efficacité des droits sociaux fondamentaux en ce qui concerne la façon dont ils ont été disposés dans le texte constitutionnel, considéré par de nombreux auteurs et même méthodologiquement inadéquates. En ce sens, il est précisé que :

Les droits sociaux fondamentaux dans la Constitution brésilienne sont loin de former un groupe homogène, parce que, en ce qui concerne son contenu et le mode de sa reconnaissance, l'Assemblée constituante n'a pas suivi ne constitué aucune ligne ou théorie précise. Au lieu de cela, fini par créer un chapitre très contradictoires au sujet de la relation interne des droits et garanties. Cela, sans doute, comporte un certain 
nombre d'inconvénients interprétatives, qui concernent directement l'efficacité des dispositions contenues dans la Constitution. (KRELL, 2002, p. 21)

Bien que, il y a cette confusion, pour Ana Paula de Barcellos, l'efficacité juridique de la norme est directement liée à la fundamentalidade sociale, comme indiqué cidessous :

Le premier critère qui guide l'identification des modalités d'efficacité juridique les déclarations réglementaires concernant vous peuvent style de condition sociale fundamentalidade car il a gouverné, qui est votre niveau d'importance ou de la pertinence sociale. C'est le paramètre logique qui anime la politique législative générale. La plus fondamentale pour la société agit sanctionnés par l'appareil et, par conséquent, les effets qu'il a l'intention de produire, plus cohérente doit être l'efficacité juridique liée (...). (BARCELLOS, 2002, p. 136)

Par efficacité juridique, entend « capacité (potentiel) d'une norme constitutionnelle de produire des effets juridiques ». (KRELL, 2002, p. 39).

Pour produire les effets juridiques, l'efficacité juridique des droits sociaux, sont dans la pleine efficacité des normes subdivisent, effectivement contenaient et peu efficace, tel qu'expliqué ci-dessous.

Normes d'efficacité complète sont appliquer de façon immédiate et sont donc indépendantes de la législation ultérieure pour son application intégrale. Il est précisé que :

Sont ceux qui, depuis l'entrée en vigueur de la Constitution, produisent ou éventuellement produire tous les effets essentiels, en ce qui concerne les intérêts, les comportements et les situations, le législateur constituant, en direct et sur le plan normatif, pour but de réglementer. (SILVA, 2005, p. 101)

Les normes constitutionnelles d'efficacité déjà contenues sont qui ont caractère obligatoire, mais limitent également de la puissance publique, tel qu'il apparait : 
Les normes d'efficacité contenues sont ceux que le législateur constituant régie suffisamment les intérêts relatifs à une affaire particulière, mais salle de gauche la partie restrictive de la compétence discrétionnaire des pouvoirs publics, conformément à la loi en établissant ou en termes de concepts généraux en elle. (SILVA, 2005, p. 116)

Et enfin et surtout, les normes d'efficacité limitée qui s'appuient sur le droit de les réglementer. Sont aussi qualifiés de "normes application différée, à celles des normes efficacité immédiate » (cellules sanguines, 2010, p. 146)

Ainsi, vous pouvez voir clairement que l'applicabilité des droits sociaux est identique à celle des garanties et des droits fondamentaux. Autrement dit, avoir applicabilité immédiate comme a le $\S 1$ de l'art. 5 du CF/1988.

\section{LA QUESTION DE L'EFFICACITÉ DES DROITS SOCIAUX DANS LA CONSTITUTION}

Beaucoup de discussion sur la doctrine sur l'efficacité et l'applicabilité des droits constitutionnels. Cependant, quelle que soit la forme de reconnaissance, les standards des droits fondamentaux doivent générer un minimum d'effets juridiques, parce que comme vous l'avez vu, tous les standards a l'efficacité et l'applicabilité.

Cependant, est que le degré d'efficacité des droits sociaux fondamentaux sera déterminé par la forme de la Constitution et la reconnaissance des particularités de votre objet. (SARLET, 2007, 237-238)

Le plus gros problème actuellement ne pas se référer aux droits, mais sur le manque d'efficacité des règles constitutionnelles, voir :

Le problème fondamental en ce qui concerne les droits de l'homme, aujourd'hui, n'est pas tant de les justifier, mais pour les protéger (...). II n'est pas une question de savoir quoi et comment sont ces droits, que la nature et sa fondation, qu'ils soient naturels ou historiques des droits, absolu ou relatif, mais ce qui est le plus sûr moyen de leur 
garantir, pour empêcher, malgré les solennelles déclarations qu'ils sont sans cesse violés (BOBBIO, 1992, p. 24-25)

De cette façon, l'efficacité réduite du social fondamental des droits non seulement l'absence de lois ordinaires.

Le plus gros problème n'est pas la fourniture de services sociaux de base par le gouvernement, puisque la grande majorité des normes pour l'exercice des droits sociaux existe déjà. Voir si le problème est dans la « formulation, mise en œuvre et l'entretien de leurs politiques publiques et dans la composition des dépenses dans les budgets de l'Union, les États et les municipalités ». (KRELL, 2002, p. 31-32)

Un autre problème qui traite de la doctrine est sur la détermination des éléments constitutifs des droits sociaux fondamentaux qui se connecte au fait de ces droits n'existent que "lorsque les lois et les politiques sociales la garantie. En d'autres termes : c'est le législateur ordinaire qui crée et détermine le contenu d'un droit social « (CANOTILHO, 2002, p. 481).

Cette partie importante de la doctrine considère que la plupart des dispositions constitutionnelles concernant les droits sociaux "sont incapables de présenter toute autre efficacité juridique en plus de lier négativement le législateur à agir explicitement contre l'objectif indiqué par la norme". (BARCELLOS, 2002, p. 162)

Cela dit, il semble qu'une grande partie de la doctrine généralement classer définissant les normes des droits sociaux comme normes programmatiques, depuis, ils nécessitent une réalisation législative qui peut générer la plénitude de ses effets.

En ce sens, il est dit que les normes de programmation sont :

Ceux à l'Assemblée législative, constituant ou non, au lieu de modifier la règle juridique d'application pratique, que des traces directeurs de lignes, qui guideront les pouvoirs publics. La législation, de la mise en œuvre et de la Justice seront subordonnée à ces préceptes, qui ressemblent à des données de programmes à votre fonction (BARROSO, 2002, p. 115) 
Pourtant, selon Krell (2002, p. 27-28), les normes de programmation servent d'alibi pour créer une image qui répond de l'État, d'un point de vue normatif, les problèmes de la société, voir :

Plusieurs normes constitutionnelles programmatique de droits sociaux, pour ne pas avoir un minimum de conditions pour sa mise en œuvre, servent seulement un alibi pour créer l'image d'un État qui répond sur le plan normatif, les problèmes réels de la société, jouant une fonction principalement idéologique constituent une forme de manipulation ou d'illusion qui immunise le système politique contre d'autres alternatives.

Toutefois, cela ne semble pas être la tendance actuelle de l'effectivité des droits sociaux, contre ces arguments, vient révéler le danger si vous laissez la volonté du législateur ou de l'administrateur de déterminer les effets sociaux totales des droits fondamentaux.

Comme États Barcellos (2002, p. 192): " cela pourrait provoquer un véritable vide le fundamentabilidade du principe de dignité de la personne humaine comme un vecteur dans l'interprétation de la Constitution. ”

Il convient de souligner ici, que la situation se complique lorsque le gouvernement maintient inerte, c'est-à-dire où pas encore installé les services nécessaires ou où travaillent mal.

Les retombées positives des droits sociaux doivent être fournies par l'État et pris en charge par la société, qui portera ces coûts, recueillies par le biais de taxes entre autres comme pris en charge:

Certainement plus clair, il y a des ressources, et les formes textuelles précises ne sera pas en mesure de surmonter cette réalité des faits, sera règles impraticables. Luis Roberto Barroso a déjà identifier cette situation, dans laquelle l'absence manifeste de conditions matérielles condamne la norme depuis sa création, comme une forme d'hypocrisie. Que se passe-t-il si vous voulez mettre l'accent sur, qui est donc, en prenant soin de l'interprétation du droit public en général, et de la Constitution 
particulière, il faut avoir à l'esprit, en plus des éléments purement juridiques, données de la réalité, un d'entre eux étant les conditions matérielles et de réaliser les contrôles réglementaires financières. (BARCELLOS, 2002, p. 259-260)

Toutefois, avant que la panne ou même un manque de services essentiels au bienêtre de la population, la "formulation de politiques publiques de protection de certaines catégories sociales économiquement marginalisés et exclus", ces politiques qui exigent principalement la gestion d'État, étant d'une importance capitale pour sa mise en œuvre, les partenariats avec la société civile. (KRELL, 2002, p. 34)

Bientôt, les droits dérivés aux avantages découlant du règlement sur les droits sociaux établis par la Constitution, implique, où déjà déployé le service public nécessaire à la satisfaction d'une fondamentale à droite, que ce n'est pas disposition en violation du droit commun peut, au Brésil, être attaquée au moyen de l'injonction, instrument juridique efficace sur le contrôle juridictionnel des actes administratifs.

Ainsi, il est précisé que :

L'efficacité qui doit accompagner les règles des droits sociaux fondamentaux - surtout quand fondée sur la dignité de la personne humaine - doit être positif ou symétrique, car il permet à l'appareil judiciaire, s'il y a une violation inconstitutionnelle, exécution de la Loi de. Les modalités d'interprétation et de l'efficacité négative vedativas de marche arrière, bien que tout à fait pertinentes et actuelles, sont une protection insuffisante de certaines variables factuelles découlant de l'impératif du respect de l'essentiel des droits sociaux. (BARKER, 2011, p. 102-103)

Pour cette raison, pour faire face aux problèmes de l'efficacité des droits sociaux fondamentaux, il est impossible de ne pas tenir compte de leur fonction première la droits de disposition, ainsi que sa reconnaissance dans le texte constitutionnel, depuis les deux aspects, tous les éléments de preuve, constituent des facteurs étroitement liés pour le degré d'efficacité et l'applicabilité des droits fondamentaux, comme déjà mentionné. 
Un autre problème qui compromet l'efficacité des droits fondamentaux est la réserve dite "possible ", ce qui a été utilisée pour indiquer la limitation des ressources avant la nécessité pour la garantie des droits fondamentaux et sociaux. Comme le démontre Barcellos (2002, p. 261) :

Le débat sur cette question a été identifié au Brésil par le biais de l'expression possible de la réservation et popularisé en grande partie par le gouvernement de l'engagement à faire la promotion et il soutient les demandes des plus divers, sous prétexte d'imminente Apocalypse économique toujours.

II s'avère que la pénurie des ressources budgétaires ne peut être des obstacles pour la garantie des droits sociaux, qui sont des conditions essentielles de la vie humaine, parce que de cette façon, en fin de compte violer un précepte fondamental de la Constitution fédérale : le principe de la dignité humaine.

De cette façon, afin de garantir le minimum existentiel est une condition nécessaire pour la survie de l'individu, parce que si vous n'avez pas les conditions nécessaires à une vie digne, les fonctions sociales ne sont pas en vigueur. Et quand il s'agit au minimum existentiel destiné à la santé, viole le droit à la vie, parce que sans la santé vous ne pouvez pas vivre.

\section{LE PRINCIPE DE RÉSERVATION POSSIBLE}

Vous avez sans doute que la garantie légale des droits sociaux ne dépend pas seulement des effets positifs de l'État, parce que leur efficacité dépend des ressources de l'État (Union, État, District fédéral et les municipalités), mais celles-ci sont limitées. Pour cette raison, l'efficacité de ces droits est limitée aux ressources budgétaires disponibles. Selon Sarlet et patron (2007, p. 265) :

Le placement de droits sociaux, aux prestations prévues par ce qu'on appelle une réserve possible, comprise au sens large, couvrant les deux la possibilité, comme le pouvoir de disposition de la part de l'acquéreur de la norme. 
Pour ouvrir une parenthèse sur le sujet, il faut tout d'abord pour conceptualiser la possible réserver Note :

La réserve de terme possible tente d'identifier le phénomène économique de la limitation des ressources disponibles sur la nécessité presque toujours infinie à remplir par eux. Ce qui compte dans l'étude menée ici, les moyens que, outre les discussions juridiques sur ce qui peut être attendu dans la juridiction de l'État - et le dernier examen de la société, car il est-ce qui nourrit - il est important de se rappeler qu'il y a une limite des possibilités matérielles de ces droits. (BARCELLOS, 2002, p. 261-262)

Cette question de la disponibilité effective des fonds est considérée par la doctrine comme une limitation factuelle à l'efficacité des droits sociaux. Cependant, il existe également une autre limite qui est de la possibilité juridique pour la disposition des ressources.

Sur le sujet, a déclaré Barcellos (2002, p. 262-263) :

Sous le titre général de la réserve peut vivre au moins deux espèces différentes des phénomènes. Le premier de ces accords avec l'absence de ressources factuelles, quelque chose proche du budget et l'épuisement peut être identifié comme une réserve de possibles factuel. II est possible de remettre en question la réalité de ce genre de circonstance en ce qui concerne les pouvoirs publics, au vu de la forme de collecte de fonds et la nature des billets. En tout cas, le manque de ressources devrait décrire des situations dont on peut parler de possible factuel réservés.

Le second phénomène identifie une réserve légale possible car il n'est pas décrit exactement un état d'épuisement des ressources et l'absence d'autorisation de budget pour certaines dépenses en particulier.

Bientôt, le début de la réserve est une limitation de fait et de droit qui va de l'état de l'aide fournissent des droits sociaux à qui de droit. C'est le gros problème soulevé ici, car il se réfère à plusieurs demande par le gouvernement de ce principe, que pour éviter l'obligation de mise en œuvre des droits fondamentaux. 
En ce qui concerne un tel problème est indiqué :

Le Brésil a l'un des pires photos de répartition des revenus dans le monde, et condition de la réalisation des droits économiques, sociaux et culturels à l'existence des ressources disponibles dans l'État, réduisant ainsi son efficacité à zéro, des moyens mis à leur universalité, les condamnant à examiner des droits mineurs (KRELL, 2002, p. 54)

Autrement dit, la majorité de la population brésilienne qui est pauvre, c'est-à-dire, déficients dans les services sociaux, c'est qui d'autre souffre de ce choc des être avoir l'exécution des droits fondés sur la disponibilité des ressources publiques.

Par conséquent, bien que l'efficacité des droits sociaux dépendent de la considération du gouvernement dans leur choix face à la rareté des ressources, le même ne peut pas se dérober son obligation constitutionnelle de fournir et d'assurer des droits minimaux à la population pour une vie digne et en l'absence de le quelconque d'entre eux, c'est à la justice, par le biais de provocation s'installer n'importe quel problème sur le sujet et qui s'est produite avec la fréquence qui montre l'analyse du problème.

\section{CONSIDÉRATIONS FINALES}

En supposant que les besoins de la population sont infinies et les ressources limitées, il convient de garder à l'esprit sur la façon d'établir des priorités dans l'affectation des ressources pour assurer le minimum établi par la Constitution fédérale.

Tout d'abord, faire remarquer que la formulation de la notion existentielle minimale, originaire de la Cour constitutionnelle allemande, qui a attiré le droit à un minimum de l'existence du principe de la dignité humaine (Constitution, art. 1, j'ai) et du droit à la vie et l'intégrité physique, à travers l'interprétation systématique par le principe de l'État Social (LF, art. 20, J'AI) (KRELL.2002.

Compléter la compréhension : 
Au premier, la qualification, qualification de la dignité humaine comme un principe fondamental exprimant la certitude que l'art. 1er, Inc. III de notre loi fondamentale ne contient-elle pas seulement une déclaration de contenu éthique et morale (qu'elle a, mais en dernière analyse, il a), mais qui constitue la norme juridique positif statut constitutionnel et, comme tel, avec efficacité, en tournant ce chanceux, au-delà de la dimension éthique ont déjà faite, à la valeur juridique de la communauté. Dans ce contexte, il faut tenir compte, autant que le principe fondamental, la dignité de la personne humaine n'est pas seulement valeur de repère des droits fondamentaux, mais de l'ordre constitutionnel ensemble, qui justifie pleinement de sa caractérisation comme un principe constitutionnel d'une plus grande axiologique hiérarchie évaluative. (SARLET, 2007, p. 111-112)

Autrement dit, le minimum existentiel est lié à la dignité de la personne humaine et doit être protégé par les droits sociaux de fonctionnalités afin de fournir des conditions minimales de la population.

Pour tenter de délimiter le contenu du minimum existentiel, une solution possible est l'utilisation du principe de dignité de la personne humaine pour assurer les conditions minimales de la population pour une vie saine ", ce qui suggère des paramètres d'orientation comment minimum établi par l'Organisation mondiale de la santé. (SARLET, 2006, p. 47-59)

Pourtant il y a toujours grande chambre pour la politique du législateur, puisqu'il n'y a pas " une mesure juste ou une façon unique de respect de l'impératif constitutionnel ", IE: fixe la teneur du droit à une existence décente mínimopara, qui ne peut être définie que dans le cas précis. (ANDRADE, 2002, p. 27)

De toutes sortes, la valeur par défaut est minimal pour la survie doit toujours inclure une base et efficace des soins de santé, accès à la nourriture de base et de I'habillement, l'enseignement du premier degré et la garantie d'un logement, comme ci-dessous : 
Maintenant, si la société n'est pas capable de reconnaître à quels point les gens se trouvent dans une situation indigne, c'est-à-dire s'il n'y a pas de consensus concernant le contenu minimum de dignité, " connaîtront une crise éthique et morale de telles proportions que le principe de la dignité humaine se transformera en une formule totalement vide » (BARCELLOS 2002, p. 197)

Bientôt, le minimum social garanti dans les conditions matérielles d'existence se fonderait sur le concept de dignité humaine et la rhétorique de ce depreciaria des droits sociaux minimaux, mais plutôt, renforcerait dans leur dimension essentielle en tant qu'expression de la citoyenneté vindicatif.

Garanti par la Constitution, le minimum existentiel doit être traitée en priorité, dit Barcellos (2002, p. 268) :

Si c'est le cas et signifie que les ressources financières ne sont pas illimités, les ressources disponibles doivent être appliqués en priorité au service des objectifs jugés essentiels par la Constitution, jusqu'à ce qu'ils sont atteints.

Les ressources restantes seront conçus en fonction des choix politiques cette délibération démocratique d'établir à chaque instant.

Tandis que les minimales existentielle, inhérente garanties constitutionnelles priorités (caractéristiques de droits fondamentaux), a une applicabilité immédiate pour être dans les droits fondamentaux indépendamment du social ou non. D'autre part, que l'alimentation ne s'occupe pas des droits sociaux liés au minimum existentiel, sera conditionné les limites des ressources budgétaires.

Cependant, c'est les pouvoirs publics un devoir de se conformer à ce que la Constitution garantit que Krell (2002, p. 60) :

Là où l'État crée ces offrandes pour le collectif, il doit s'assurer de la possibilité de la participation citoyenne. Et si la loi n'accorde pas le droit d'exprimer les services vitaux récepteurs individuels, le citoyen peut faire appel au droit fondamental de l'égalité dans le cadre du principe de l'État Social 
Une fois violé le minimum existentiels, par l'omission de l'efficacité de la social fondamentale des droits, le droit de demander à la Cour.

La raison qui justifie cette judiciarisation des droits sociaux, inhérentes au minimum existentiel, en vertu de laquelle ces droits sont indispensables pour avoir une vie avec dignité, qui est la théorie de la réserve ne peut pas soumettre ici. Bien que judiciarisation essayant de diminuer ce problème de manque de ressources, vous devez avoir une vue sur l'objectif essentiel, prévues par la Constitution, et si les ressources sont suffisantes pour atteindre la destination. (BARKER, 2011).

Mais se trouve confronté le minimum existentiel qui permettra d'évaluer les priorités pour les dépenses publiques, conformément à la Constitution et aussi peut définir les activités juridictionnelles des droits sociaux

Selon Canotilho (2002), l'État doit assurer l'efficacité des droits sociaux, comme vous le savez déjà, mais il convient de dire, trop, que le même ne peut pas réduire, annuler ou révoquer ce qui aujourd'hui nous comprenons comme minimum existentiel et doivent sceller toutes les mesures législatives visant à cela. C'est le principe de l'interdiction de la réaction sociale.

Conceptualisation-if l'inverse dit :

L'inverse, enfin, est une dérivation de l'efficacité négatif, liée en particulier aux principes qui concernent les droits fondamentaux. Elle part du principe que ces principes sont mis en œuvre par le biais de normes infra-constitutionnelles et qui, fondée sur le droit constitutionnel en vigueur, un effets généraux visés par ces principes est l'expansion progressive des droits fondamentaux. À partir de ces hypothèses, que l'inverse peut nécessiter la magistrature propose est la nullité, l'annulation de la norme qui, en régulant le principe, pour accorder ou d'étendre les droits fondamentaux, sans laquelle la révocation en question est accompagnée d'une politique de remplacement ou l'équivalent. (BARROSO, 2002, p. 379)

Il est donc conclu que ce cachet est un moyen de prévenir l'abrogation des lois garantissant des droits sociaux, ne pas à diminuer ce qui a été réalisé par le biais de 
l'évolution historique, telles que l'établissement de normes minimales afin d'avoir une vie digne.

De cette façon, le rôle des juges en matière de droits sociaux est important parce que c'est le contrôle des droits sociaux fondamentaux, analysant le caractère essentiel de la demande et le degré de nécessité selon chaque cas individuel. Et que ces "judiciarisée" droits sociaux et en particulier la santé, objet de la présente étude, on remet la judiciarisation du phénomène de la santé.

\section{RÉFÉRENCES BIBLIOGRAPHIQUES}

ALMATY. Alma-Ata. 1978. Disponible à: http://www.saudepublica.web.pt/05promocaosaude/Dec_Alma-Ata.htm. Accès au : 09 nov. 2015.

ANDRADE, de José Carlos Vieira. Le pouvoir judiciaire et les droits fondamentaux sociaux : accès à la santé, la controverse de la question. Porto Alegre : La Cour de Justice, 2002.

BARCELLOS, Emanuel. L'efficacité juridique des principes constitutionnels : le principe de la dignité humaine. Rio de Janeiro : Renovar, 2002.

BARROSO, Luís Roberto. Le droit constitutionnel et l'efficacité de ses règles : limites et possibilités de la Constitution brésilienne. 6 ed. São Paulo : Renouveler, 2002

BARKER, Ramon F. Judiciarisation du droit à la santé : la tension entre le " minimum existentiel » et la "réserve possible » dans la recherche de la préservation de la dignité humaine. Curitiba : Juruá Editora, 2011.

Brésil. Constitution (1988). Constitution de la République fédérative du Brésil. Brasilia. DF: Sénat, 2014.

Brésil. Loi n 8080 du 19 septembre 1990. Règles sur les conditions de la promotion, la protection et le rétablissement de la santé, organisation et fonctionnement des 
services correspondants et autres questions. Diário Oficial da União, pouvoir exécutif, Brasilia, DF.

GLOBULES, Uadi Lammego. Droit constitutionnel à la portée de tous. 2eéd. São Paulo : Saraiva, 2010.

CANOTILHO, José Joaquim Gomes. Droit constitutionnel et théorie de la Constitution. 6. ed. Coimbra : Almedina, 2002.

KRELL, Andrea Joachim. Droits sociaux et le contrôle judiciaire au Brésil et en Allemagne : les chemins de (dé) d'un droit constitutionnel comparé. Porto Alegre : Sergio Antonio Fabris, 2002

Moraes, Guilherme pitié. Cours de droit constitutionnel. São Paulo : Atlas, 2014.

SARLET, Wolfgang Ingo. L'efficacité des droits fondamentaux. 7. Ed. Porto Alegre : Editora avocat, 2007. . La dignité humaine et des droits fondamentaux dans la Constitution fédérale de 1988. 4 ed. Porto Alegre : Editora avocat, 2006.

SILVA, José Afonso da. Commentaire de contextuels sur la Constitution. São Paulo : Malheiros, 2005. 